\title{
Light interference pattern measurements from automated low-cost Young's double-slit experiments
}

\author{
C. G. de Oliveira Jr ${ }^{1}$, M. R. Gumiero ${ }^{1}$, V. R. Coluci* ${ }^{*}$ \\ ${ }^{1}$ Universidade Estadual de Campinas, Faculdade de Tecnologia, 13484-332, Limeira, SP, Brasil
}

Received on January 02, 2020. Revised on February 10, 2020. Accepted on February 10, 2020.

\begin{abstract}
Young's double-slit experiment is commonly used in undergraduate physics laboratory to show the wave nature of light. As an alternative to expensive commercial kits, here we present an automated, low-cost light intensity measurement setup that allows real-time visualization of the interference pattern. The light intensity is measured by a light dependent resistor placed on the head of a dot matrix printer. The head motion is controlled by an Arduino which also collects the data and send them to a computer. The interference pattern obtained by the setup is in agreement with a model that includes the degree of the coherence of the light source and the effective size related to the light collecting area of the resistor. The apparatus allows quantitative data analysis from the measured light patterns and is suitable to be used in undergraduate physics laboratories.
\end{abstract}

Keywords: double slit, light waves, Arduino, low cost, light interference.

\section{Introduction}

Considered as one of the ten most beautiful experiments in science 1, 2], the classical Thomas Young's double-slit experiment proved the wave nature of light. In Young's Course of Lectures on Natural Philosophy and the Mechanical Arts of 1807 [3], he applies the principles of interference in waves of water and in waves of sound to light and describes the double-slit experiment ( [3], p. 464)

"In order that the effects of two portions of light may be thus combined, it is necessary that they be derived from the same origin, and that they arrive at the same point by different paths, in directions not much deviating from each other. This deviation may be produced in one or both of the portions by diffraction, by reflection, by refraction, or by any of these effects combined; but the simplest case appears to be, when a beam of homogeneous light falls on a screen in which there are two very small holes or slits, which may be considered as centres of divergence, from whence the light is diffracted in every direction."

and its results (3, p. 464-465):

"when the two newly formed beams are received on a surface placed so as to intercept them, their light is divided by dark

*Correspondence email address: vitor@ft.unicamp.br stripes into portions nearly equal, but becoming wider as the surface is more remote from the apertures, so as to subtend very nearly equal angles from the apertures at all distances, and wider also in the same proportion as the apertures are closer to each other."

Besides the importance on the establishment of the wave nature of light, the double-slit experiment was further used to develop the concept of degree of spatial coherence of light [4,5]. This concept has been used, for instance, to determine the coherence properties of manmade ultra-violet [6], ultra-short X-ray [7], and visible [8] light sources. Recently, different apparatus have been proposed to bring the concept of spatial coherence to undergraduate physics laboratories $[9,10]$. The degree of coherence has been applied in stellar interferometry [11,12 to determine the size of astronomical objects. This is usually done by relating the degree of spatial coherence with the size of the light source through the van Cittert-Zernicke theorem [4,13].

The double-slit experiment is commonly used in physics courses to show the wave nature of light through the observation of the interference pattern on a screen. Knowing the wavelength of the incident light and the distance between the screen and the slits, the students can also determine the separation between the slits by measuring the location of the interference maxima. These measurements are usually made with a ruler directly on the screen. More precise measurements can be achieved by sophisticated, automated systems from expensive commercial kits ( US\$1000), that include a laser source, a 
diffraction mask with the slits, a track, and a software for data collection and analysis. For example, some common commercial diffraction and interference apparatus can be obtained from Vernier (US\$ 850) 14 and from PASCO (US\$ 1130) 15]. Because the whole interference pattern is obtained from the data collection system, fittings and comparisons with the theoretical predictions can be easily carried out. Alternative to those expensive kits, low-cost schemes have been proposed to scan diffraction patterns which include, for instance, flatbed scanners [16], XY recorders [17], stepper motors coupled with a screw [18], photodiode coupled with a handsaw blade and an oscilloscope [19], and charge-coupled device detectors [20].

In this work, we provide an alternative, low-cost $(\sim$ US\$ 100), automated apparatus to quantify the interference patterns produced in the double-slit experiment. The apparatus is controlled by an Arduino and is comprised of a green laser pointer as the laser source, parts of a dot matrix printer, and a light dependent resistor as the intensity acquisition system. The collected intensities are shown in real-time on a screen of a computer integrated with the apparatus. The apparatus allows quantitative data analysis from the measured light patterns and reasonable agreement with theoretical predictions. Because all the parts of the apparatus can be accessible to the students, the apparatus can be considered a 'transparent gadget' 21] which provides the students opportunities to physically interact with the apparatus and to discuss possible improvements. Such transparent gadget, in contrast to black-box technologies, can promote curiosity and motivate active learning in the classrooms and can improve the data analysis of other related optical experiments 22 .

\section{Model}

The interference intensity produced by a monochromatic, totally coherent light of wavelength $\lambda$ as a function of

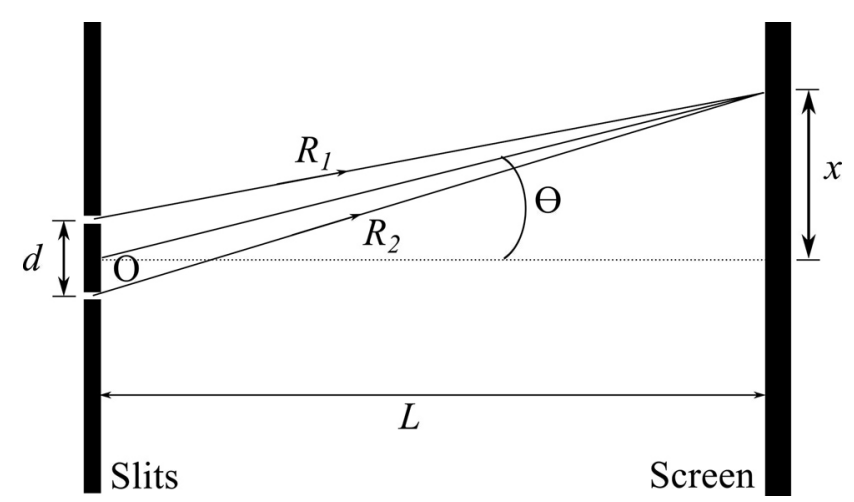

Figure 1: Interference of two light beams $R_{1}$ and $R_{2}$ on a screen in the double-slit experiment. angle $\theta$ (Fig. 1) is given by 10

$$
I(\theta)=I_{0} \operatorname{sinc}^{2}\left(\frac{k a \sin \theta}{2}\right) \cos ^{2}\left(\frac{k d \sin \theta}{2}\right),
$$

where $I_{0}$ is the maximum intensity, $a$ is the slit width, $d$ is the separation between the slits, $k=2 \pi / \lambda$ is the magnitude of the wave vector, and $\operatorname{sinc}(\alpha) \equiv \sin (\alpha) /(\alpha)$. The term involving the sinc function is related to the diffraction of each slit due to their finite widths which modulate the interference pattern.

When the distance between the slits and the detection screen $L$ is large and $L \gg d$ (Fraunhofer limit), the far-field conditions are satisfied and the approximation $\sin \theta \sim \tan \theta \sim x / L$ can be used (Fig. 1). Thus, the equally spaced intensity maxima are located at

$$
x_{n}^{\max }=\frac{\lambda L}{d} n, \quad n=0,1,2, \ldots \ldots
$$

The finite size and the spectral bandwidth of the light source can reduce its coherence (temporal or spatial). For partially coherent light sources, the visibility of the interference fringes decreases 23. and Eq. (1) is written, in the Fraunhofer limit, as

$$
I(x)=\frac{I_{0}}{2} \operatorname{sinc}^{2}\left(\frac{k a}{2 L} x\right)\left[1+V \cos \left(\frac{k d}{L} x+\delta\right)\right],
$$

where $V$ is the fringe visibility which is related to the spatial coherence of the source. If the light source is complete coherent $(V=1)$, Eq. (3) reduces to Eq. (1). On the other hand, for a complete incoherent light $(V=0)$ as the one from natural sources, no interference pattern is observed and $I(x)$ is just the sum of the intensities originated from each slit. The phase $\delta$ is introduced to describe the cases when the light source resides off axis at $x \neq 0$.

\section{Apparatus}

Our apparatus consists of three main parts: the light source and the slits; the intensity data collector; and the controller hardware and software. A scheme indicating the main parts and some photographs of the apparatus are shown in Fig. 2.

\subsection{Light source and the slits}

We used a green laser pointer (laser class $3,<5 \mathrm{~mW}$ ) of wavelength $532 \pm 10 \mathrm{~nm}$ as the light source (Fig. $2 \mathrm{~b}$ bottom). The laser pointer's on/off button was removed and the batteries were replaced by an external, stabilized power supply (0-10 V, 2 A) to control the laser intensity (Fig. $2 \mathrm{~b}$ bottom). Because the quality of the interference pattern is influenced by the quality of the slits, we used a diffraction mask produced by electron-beam lithography with a double-slit with $a=36 \pm 2 \mu \mathrm{m}$ and $d=248 \pm 5 \mu \mathrm{m}$ (Fig. 2b). Alternatives to the present homemade diffraction mask can be used in the apparatus. For example, 


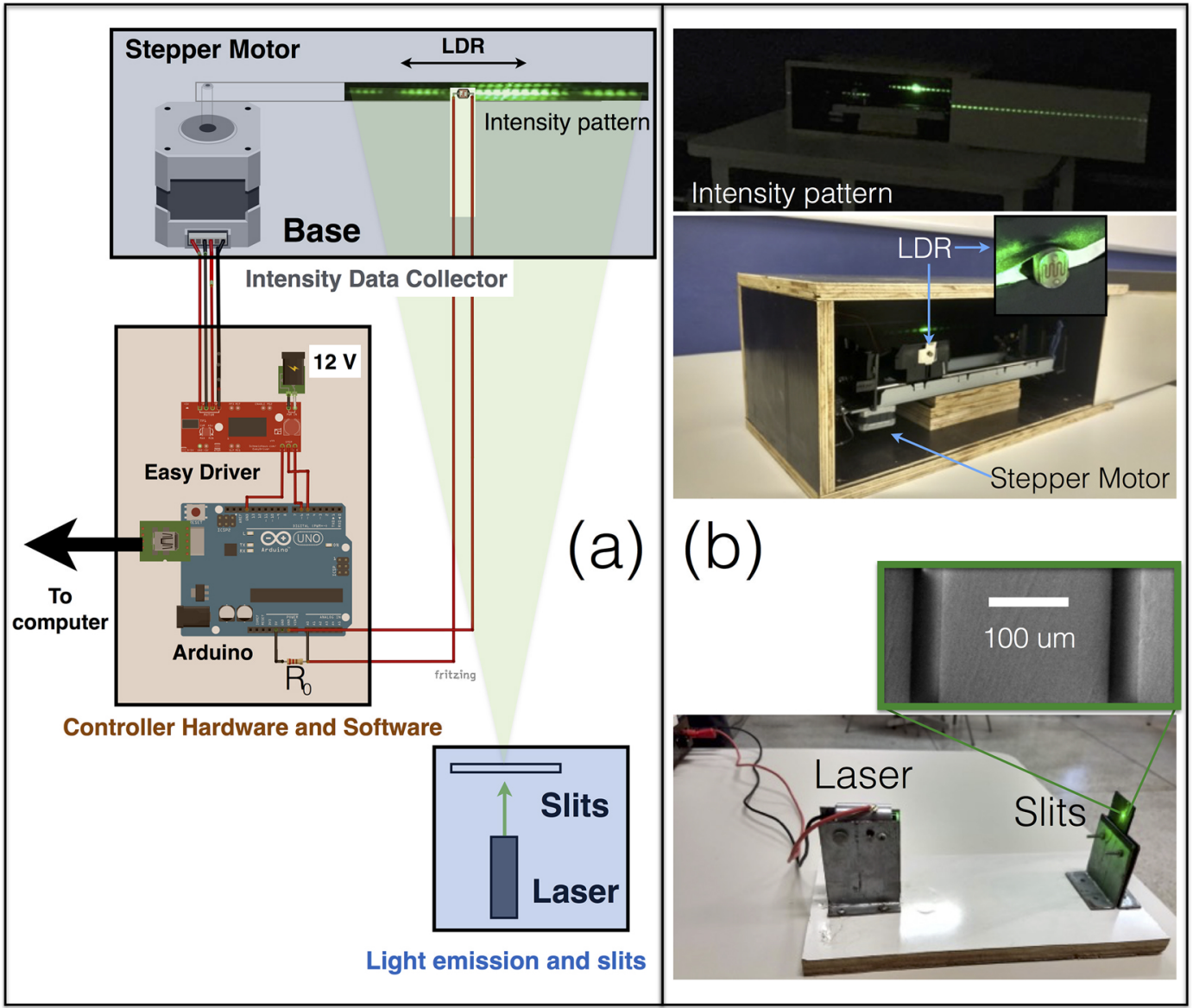

Figure 2: (a) Schematic representation of the three main parts of the apparatus. The LDR and the resistor $R_{0}(=10 \mathrm{k} \Omega)$ are in series. (b) Photographs of the apparatus showing (bottom) the laser beam reaching the slits, (middle) the wood base for the printer carriage, the LDR, and the stepper motor, and (top) an example of an obtained intensity pattern. A scanning electron microscopy image of the double-slit used in this work is also shown in the inset. The diagram of the circuit involving the Arduino, the Easy driver, and the stepper motor was built with Fritzing [24].

the widely available diffraction masks from PASCO 25 present double slits with $a=40 \mu \mathrm{m}$ and $d=250 \mu \mathrm{m}$, similar to the ones we used in this work. Both the laser and the diffraction mask were fixed in metallic supports (Fig. 2 bottom) to mechanically stabilize the intensity patterns. Misalignment between the laser beam and the middle position between the slits may occur and shift the central maximum from $x=0$. This shift is described by the phase $\delta$ in the cosine function of Eq. (3).

\subsection{Intensity data collector}

To collect the interference pattern produced by the laser after reaching the slits, we used a light dependent resistor (LDR, model GL5528, $5 \mathrm{~mm}$ diameter, $100 \mathrm{~mW}$ maximum power) as the photodetector. Because the LDR resistance $R$ varies nonlinearly with the illuminance $I_{l}$ as $R \sim I_{l}^{-b}$, a scale conversion was necessary to obtain the intensity from the measured voltage across the LDR. We used the LDR datasheet to estimate $b=0.7$. The laser intensity $I$ on a position $x$ on the screen was then determined as a function of the voltage across the LDR when it is located at $x\left(V_{\mathrm{LDR}}(x)\right)$

$$
\frac{I(x)}{I_{0}}=\left[\left(\frac{V_{T}}{V_{\mathrm{LDR}}(x)}-1\right)\right]^{-\frac{1}{b}}
$$

with $V_{T}=5 \mathrm{~V}$.

The choice of the LDR as the photodetector was to keep the apparatus as cheap as possible. On the other hand, the finite size of the LDR (inset Fig. 2b) limits the apparatus resolution. To overcome this limitation, we adjusted $L$ to about $6 \mathrm{~m}(625 \pm 5 \mathrm{~cm})$ so the separation between the light maxima became larger than the LDR size. Because such $L$ may be large for classrooms with limited space, we have also performed the experiment with a LDR partially covered by a black tape (Fig. 3). Whereas covering the LDR increased its spatial resolution by reducing its lateral size, this leads to smaller measured voltages. However, this procedure allowed us to obtain reasonable diffraction patterns for a length of about $2 \mathrm{~m}$ (Fig. 3). Given the LDR orientation used in the apparatus (inset Fig. 2b), the LDR size is $4.3 \pm 0.2 \mathrm{~mm}$ according to the LDR datasheet. However, we estimated the effective 


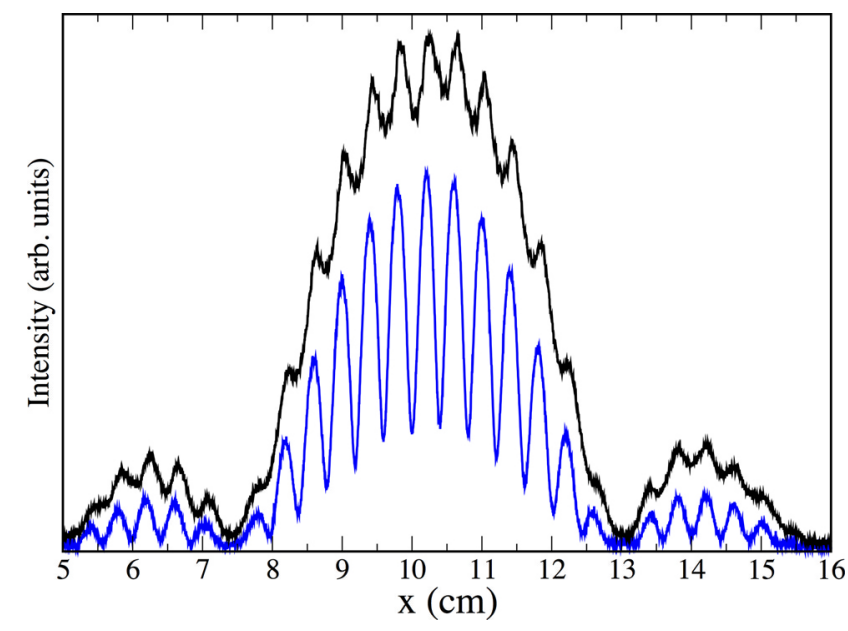

Figure 3: Raw voltage data from the uncovered (black) and partially covered (blue) LDR by a black tape for $L=208 \pm 1 \mathrm{~cm}$. The minima exhibit a non-zero intensity because neither the LDR data were averaged, to take into account the LDR lateral size, neither scaled, to take into account the nonlinear dependence of the LDR resistance with the illuminance. The LDR size after covered with the tape was about $2 \mathrm{~mm}$ which yields to a effective size of about $1 \mathrm{~mm}$.

size $l$, related to the light collecting area, to be about half of that value due to the serpentine format of the photo-sensitive track. The effect of the LDR size on the interference pattern (Eq. (3)) was estimated by averaging $I(x)$ on a window of width $l$

$$
\tilde{I}\left(x_{i}\right)=\frac{1}{n_{j}(l)} \sum_{j=1}^{n_{j}(l)} I\left(x_{j}\right)
$$

where $n_{j}(l)$ is the number of data points satisfying $\mid x_{i}-$ $x_{j} \mid<l / 2$.

The motion of the LDR along the interference pattern ( $x$ direction) was provided by a set comprised of a head, a stepper motor, and a carriage support of an unused dot matrix printer (Epson LX-300+II). The use of a stepper motor provided a precise positioning control [18] whereas the reuse of parts of the printer saved time and resources related to the apparatus fabrication and assemblage. We were able to move the LDR (left or right) by placing it on the printer head and by turning on the stepper motor (model Epson EM-326). The whole set was fixed in a wood box where the interference patterns were recorded (Fig. 2b).

\subsection{Controller hardware and software}

Finally, the stepper motor and the collection of the voltage across the LDR were controlled by an Arduino UNO board [26] (Fig. 2a). An Easy driver board was coupled with Arduino to allow controlling the motor speed and rotation (clockwise and anticlockwise) [27]. The motor was powered by a $12 \mathrm{~V}$ power supply connected to the Easy driver board. The Arduino code controls the stepper motor, collects the voltage across the LDR, calculates the average and standard deviation, and sends them to the serial port. Because of the 10-bit resolution of the Arduino anologic-digital converters, the raw values of the voltage across the LDR sent to the serial port are integers between 0 and 1023. These data can be further processed in any graphing software to determine the positions of the interference maxima and to compare with theory (Eq. (2) and (3)).

To visualize the raw voltage across LDR in real-time, we also implemented a code in the Processing language [28] to plot the voltage obtained from the serial port during data acquisition. This real-time data visualization is very useful during the experiment preparation, to fix possible misalignment between the light pattern and the base, and during the measurements, to discuss the results with the students. In this plot, the vertical axis shows the voltage across the LDR $\left(V_{\mathrm{LDR}}\right)$ in units of $V_{T} / 1023$ and the horizontal axis shows the LDR position in units of $0.028 \mathrm{~mm}$ (the distance per step of the stepper motor). The code also includes buttons to move the LDR towards the left and right directions. Both the Arduino and Processing codes are available in Github repository 29].

The cost of the apparatus can be about US\$ 50 if a dot matrix printer and a diffraction mask are available. The Arduino and Easy driver boards, LDR, power supplies, laser pointer, and wood base can be acquired by less than US\$ 50. Used dot matrix printers can be acquired very cheaply and the diffraction masks containing sets of double-slits are usually found in most optical laboratories of physics courses. If not, a typical cost of such masks is about US $\$ 150$ or less.

\section{Results}

Common measurements of the locations of the intensity maxima of the interference pattern can be easily done with the proposed apparatus. Figure 4 shows the positions of these maxima produced by the double-slit (Fig. 2b). These positions were measured from the plot of the normalized intensity as function of the LDR position (Fig. 5). Fitting the data with Eq. (2) and using $\lambda=$ $532 \pm 10 \mathrm{~nm}$ and $L=625 \pm 5 \mathrm{~cm}$, we obtained $d=278 \pm 19$ $\mu \mathrm{m}$ which is close to the predicted value from microscopy analysis. This reasonable agreement shows the apparatus can be applied to typical double-slit experiments conducted in physics courses to determine the separation between the slits.

Figure 5 shows the interference pattern measured from the apparatus and the predicted one by Eq. (3) $a=38 \mu \mathrm{m}$, $d=274 \mu \mathrm{m}, L=625 \mathrm{~cm}$, and $\lambda=532 \mathrm{~nm}$. These parameters were obtained by using a simple trial and error process. We considered a phase of $1 \%$ of $k d / L$ in Eq. (3) to fix the observed shift of the central peak. The predicted value for the slit width is in good agreement with the value determined by microscopy. The prediction for a 


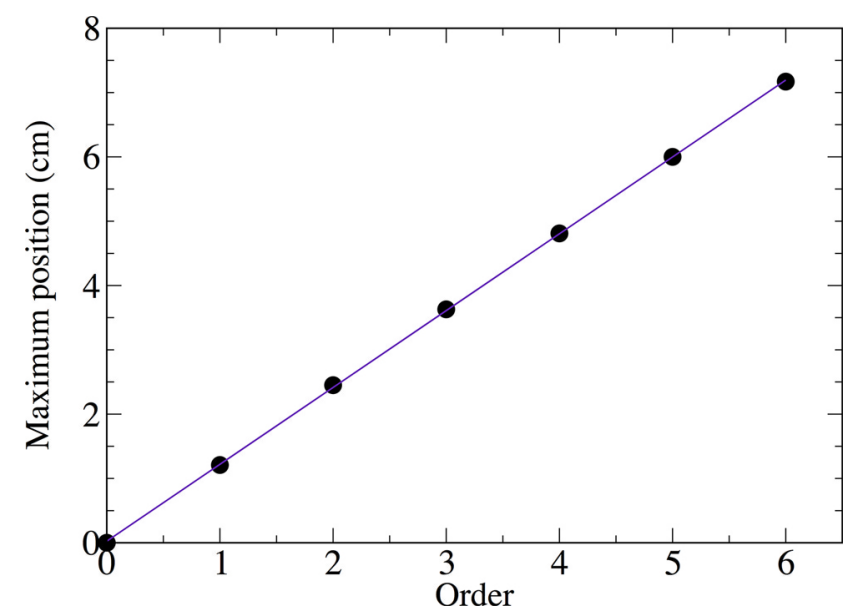

Figure 4: Locations of the interference maxima measured from the apparatus (circles). The fitting with Eq. (2) $x_{n}^{\max }($ in $\mathrm{cm})=$ $(0.02 \pm 0.02)+(1.195 \pm 0.005) n$ is also shown.

coherent light source ( $V=1$, Fig. 5a) provides a reasonable agreement but fails to describe most of regions close to the maxima and minima. The agreement is improved when a partially coherent light is assumed with a fringe visibility of $V=0.9$ (Fig. 5b). Finally, when the finite size of the LDR is also considered (Fig. 5c), maxima and minima are even well described. The exception is the central peak for which the difference with respect the theory was attributed to possible reflections from the diffraction mask that may increase the intensity at $x=0$. The curve in Fig. $5 \mathrm{c}$ was obtained for $l=2.4 \mathrm{~mm}$ which is comparable to the estimated effective size of the LDR of about $2 \mathrm{~mm}$.

\section{Conclusions}

The measurements of the light intensity on the Young's double-slit experiment was automated with a low-cost apparatus comprised of recycled parts of a dot matrix printer controlled by Arduino and a light dependent resistor as the photodetector. The locations of the intensity maxima are in reasonable agreement with theory and the interference pattern was well described by a model that includes the degree of the coherence of the light source and the effective size of the photodetector. The apparatus is an alternative to expensive commercial double-slit kits and is suitable to undergraduate physics laboratory.

\section{Acknowledgments}

We thank the financial support from the Student Support Service (SAE)/UNICAMP and the Prof. Varlei Rodrigues and Antonio C. da Costa (IFGW/UNICAMP) for providing the diffraction mask. We also thank Prof. Ivan de Oliveira (FT/UNICAMP) for helpful discussions and Prof. Luis F. de Avila (FT/UNICAMP) for the scanning electron microscope images.

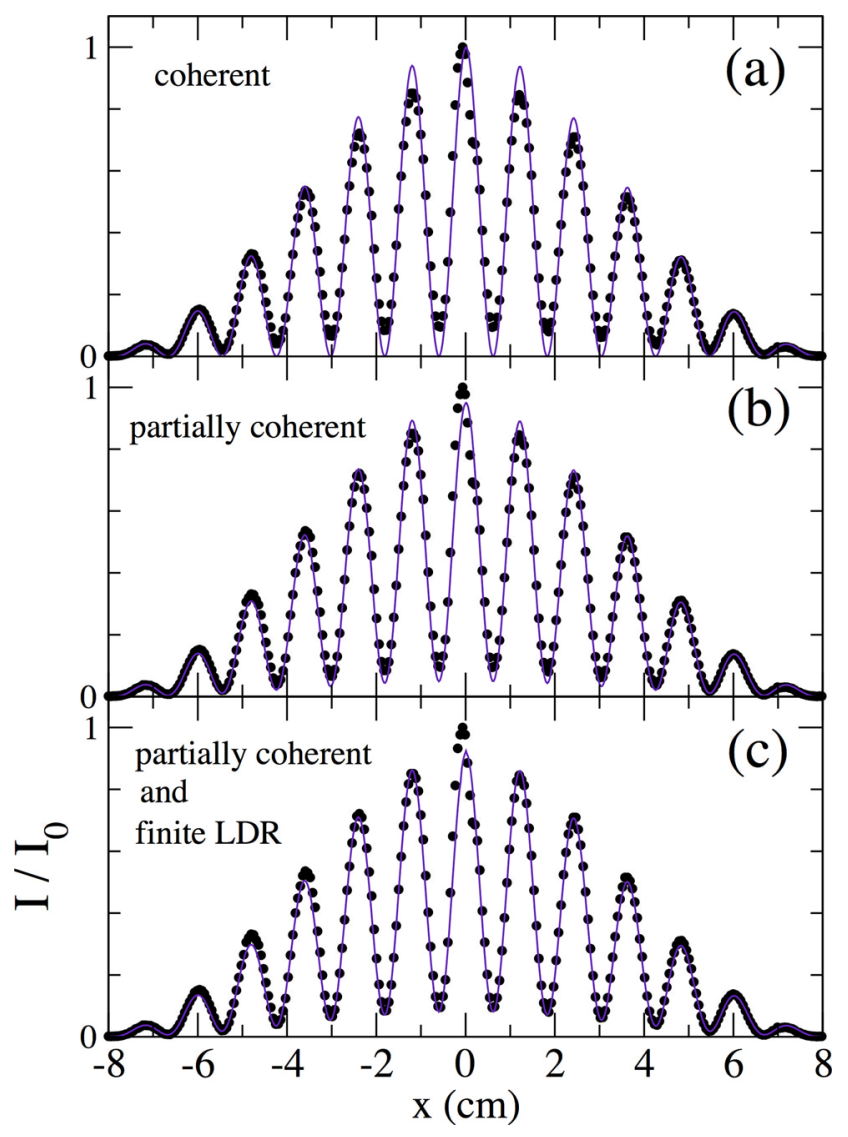

Figure 5: Comparison between the interference pattern obtained with the apparatus (circles) with the predicted one (lines) by Eq. (3) ((a) and (b)) and Eq. (4) (c). The parameters used for all curves were $a=38 \mu \mathrm{m}, d=274 \mu \mathrm{m}, L=625 \mathrm{~cm}$, and $\lambda=532 \mathrm{~nm}$.

\section{References}

[1] R.P. Crease, The Prism and the Pendulum: The Ten Most Beautiful Experiments in Science (Random House, New York, 2003).

[2] R.P. Crease, Phys. World 15, 19 (2002).

[3] T. Young, Lectures on Natural Philosophy (J. Johnson, London, 1807), v. 1.

[4] F. Zernike, Physica 5, 785 (1938).

[5] B.J. Thompson and E. Wolf, J. Opt. Soc. Am. 47, 895 (1957).

[6] C. Chang, P. Naulleau, E. Anderson and D. Attwood, Opt. Comm. 182, 25 (2000).

[7] A. Singer, I.A. Vartanyants, M. Kuhlmann, S. Duesterer, R. Treusch and J. Feldhaus, Phys. Rev. Lett. 101, 254801 (2008).

[8] S.T. Wang, D.L. Rubin, J. Conway, M. Palmer, D. Hartill, R. Campbell and R. Holtzapple, Nucl. Instrum. Meth. A703, 80 (2013).

[9] J.P. Sharpe and D.A. Collins, Am. J. Phys. 79, 554 (2011).

[10] D.P. Jackson, N. Ferris, R. Strauss, H. Li and B.J. Pearson, Am. J. Phys. 86, 683 (2018).

[11] A. Glindemann, Principles of Stellar Interferometry (Springer-Verlag Berlin Heidelberg, 2011).

[12] F. Millour, New Astronomy Reviews 52, 177 (2008). 
[13] P.H. van Cittert, Physica 1, 201 (1934).

[14] https://www.vernier.com/products/sensors/dak/

[15] https://www.pasco.com/prodCatalog/EX/EX-5545_ interference-and-diffraction-of-light-experim/ index.cfm.

[16] T. Koopman and V. Gopal, Am. J. Phys. 85, 392 (2017).

[17] http://www.ises.info/index.php/en/laboratory/ experiment/difraction-on-microobjects/ experiment

[18] http://spa-mxpweb.spa.umn.edu/resources/ ExpWriteups/LabManDiff.pdf

[19] S. Ganci, Am. J. Phys 67, 357 (1999).

[20] C. Lengacher, S. Macklin, D. Hite and M.F. Masters, Am. J. Phys 66, 1025 (1998).

[21] L. Kowalski, Physics Today 66, 8 (2013).

[22] L.A. Souza, L. da Silva, J.A.O. Huguenin and W.F. Balthazar, Revista Brasileira de Ensino de Física 37, 4311 (2015).

[23] J.E. Greivenkamp, in: Handbook of optics, edited by M. Bass (McGraw-Hill, New York, 1995), 2 ed.

[24] A. Knörig, R. Wettach and J. Cohen, in Proceedings of the 3rd International Conference on Tangible and Embedded Interaction, Cambridge, 2009, edited by A. Knorig, R. Wettach and J. Cohen (Association for Computing Machinery, New York, 2009), p. 351.

[25] https://www.pasco.com/prodCompare/diffractionoptics-kit/index.cfm

[26] http://www.arduino.cc/

[27] http://www.schmalzhaus.com/EasyDriver/ and http://www.schmalzhaus.com/EasyDriver/Examples/ EasyDriverExamples.html

[28] https://processing.org/

[29] https://github.com/vitorcoluci/double-slit/ 